\title{
Bicuspid aortic valve and ascending aortic aneurysm - genetic background of the disease
}

\author{
Justyna Rybicka ${ }^{1}$, Mariusz Kuśmierczyk², Mirosław Kowalski', Piotr Hoffman ${ }^{1}$ \\ 1Department of Congenital Heart Diseases, Institute of Cardiology, Warsaw, Poland \\ 2Department of Cardiac Surgery and Transplantology, Institute of Cardiology, Warsaw, Poland
}

Postep Kardiol Inter 2012; 8, 2 (28): 114-119 DOI: 10.5114/pwki.2012.29652

Key words: bicuspid aortic valve, aortic regurgitation, aortic aneurysm, aortic dissection, inheritance

\section{Introduction}

Bicuspid aortic valve (BAV) is the most common congenital heart disease. Its incidence in the general population is estimated at $1-2 \%[1,2]$. There are 3 anatomical types of BAV: with a fusion of the right and left coronary leaflet responsible for $74 \%$ of all cases; with a fusion of the right and non-coronary leaflet (24\%); and most rarely with a fusion of the left and non-coronary leaflet (2\%) [3]. The consequence of BAV is its stenosis and/or regurgitation and the increased risk of infective endocarditis. The defect is often accompanied by an ascending aortic aneurysm. Other congenital heart diseases co-existing with BAV are coarctation of the aorta, interruption of the aortic arch, ventricular septal defect (VSD), patent ductus arteriosus (PDA) and atrial septal defect (ASD) [1, 2, 4]. Inheritance of the disease has been demonstrated. The defect is three times more common in men than in women [1].

Below we present a case of a 27-year-old man with significant regurgitation and moderate stenosis of the bicuspid aortic valve and ascending aortic aneurysm (thoracic aortic aneurysm disease - TAAD).

\section{Case report}

At the age of 18 the heart murmur was detected and after echocardiographic examination the patient was diagnosed with aortic regurgitation. He was referred to the Department of Adult Congenital Heart Diseases of the Institute of Cardiology. At that time he was diagnosed with significant regurgitation and mild BAV stenosis (maximal aortic gradient - GA max $42 \mathrm{~mm} \mathrm{Hg}$, GA mean $30 \mathrm{~mm} \mathrm{Hg}$ ). Because of the lack of symptoms and moder- ately dilated left ventricle (left ventricular end diastolic diameter [LVEDd] $65 \mathrm{~mm}$ ) with its preserved systolic function (ejection fraction [EF] 73\%) and borderline diameter of the ascending aorta $(38 \mathrm{~mm})$ a decision was made to continue observation. Over the next 7 years the patient remained under the care of the Adult Congenital Heart Diseases Outpatient Clinic, where a gradual increase of the aortic valve gradient was observed (GA max from $42 \mathrm{~mm} \mathrm{Hg}$ to $72 \mathrm{~mm} \mathrm{Hg}$, GA mean from $30 \mathrm{~mm} \mathrm{Hg}$ to $40 \mathrm{~mm} \mathrm{Hg}$ ) accompanied by dilation of the left ventricle (from $65 \mathrm{~mm}$ to $70 \mathrm{~mm}$ ) and its progressive systolic dysfunction (EF drop from $73 \%$ to $60 \%$ ) with enlarged ascending aorta (from $38 \mathrm{~mm}$ to $46 \mathrm{~mm}$ ) (Table 1). At the age of 25 the patient noticed worsening of exercise tolerance. Echocardiographic examination demonstrated significant dilation of the left ventricle (LVEDd $70 \mathrm{~mm}$ ) with impaired systolic function (EF 50\%) and significantly enlarged ascending aorta $(50 \mathrm{~mm})$. The patient was urgently referred to the Department of Congenital Heart Diseases, but he did not report for the next 2 years. He was not hospitalized until the age of 27 years. At that time the patient's subjective physical performance was assessed as New York Heart Association (NYHA) class II/III. Physical examination revealed arterial pressure of 140/78 mm Hg, Corrigan's pulse, systolic thrill in the $2^{\text {nd }}-3^{\text {rd }}$ intercostal space, systolic murmur best heard in the $2^{\text {nd }}$ intercostal space (4/6 on the Levine scale) and diastolic decrescendo murmur best heard at the lower left sternal margin (3/6 on the Levine scale). Electrocardiogram (ECG) showed signs of left ventricular hypertrophy and overload (Figure 1). Chest X-ray demonstrated mild enlargement of the heart affecting the left ventricle (cardio-thoracic ratio of 52\%), enlarged ascending aorta enlargement

\section{Corresponding author:}

Justyna Rybicka MD, Department of Congenital Heart Diseases, Institute of Cardiology, 42 Alpejska, 04-628 Warsaw, Poland, tel.: +48 223434 263, fax: +48 223434 538, e-mail: jrybicka@ikard.pl

Praca wpłynęła: 27.03.2012, wersja poprawiona: 15.05.2012, przyjęta do druku: 28.05.2012. 
and normal picture of the pulmonary circulation (Figure 2). Echocardiographic examination revealed dilated left ventricle (LVEDd $72 \mathrm{~mm}$ ), significant aortic valve regurgitation accompanied by its moderate stenosis with maximal GA of $64 \mathrm{~mm} \mathrm{Hg}$ and mean GA of $41 \mathrm{~mm} \mathrm{Hg}$ and ascending aorta enlargement up to $52 \mathrm{~mm}$ (Figure 3). There was also an additional linear echo in the aortic lumen, which could correspond to the dissected intima and whose presence was confirmed by transesophageal echocardiography (Fig. 4). The echo was likely a reverberance as its motion was concordant with the heart cycle and parallel to the aortic wall, but because of the intensity of the echo and its visibility in various projections, it required further evaluation. A computed tomography examination was performed to exclude aortic dissection and to fully assess the size and morphology of the aneurysm and its relations with neighboring organs and arteries originating from the aorta. The study demonstrated ascending aortic aneurysm with maximal diameter of $52 \mathrm{~mm}$, excluded the presence of dissection and, as in the echocardiographic picture, confirmed the presence of bicuspid aortic valve with a fusion of the right and left coronary leaflet and calcifications at the margins of these leaflets (Figures 5, 6). The patient was qualified for urgent surgical treatment and underwent the Bentall procedure including implantation of an aortic prosthesis with a mechanical aortic valve (aortic valvular graft, St. Jude Medical $27 \mathrm{~mm}$ ). The post-operative course was uneventful. The patient was discharged home on day 8 after surgery. Eight months after the surgery he remains in NYHA class I/II.

\section{Discussion}

The genetic background of the disease determines the high incidence of BAV. Several ways of inheritance

Table 1. Transthoracic echocardiography, patient aged $19-25$ years

\begin{tabular}{lccccc}
$\begin{array}{l}\text { Age } \\
\text { [years] }\end{array}$ & GA max & GA mean & $\begin{array}{c}\text { LVEDd } \\
{[\mathrm{mm}]}\end{array}$ & $\begin{array}{c}\text { EF } \\
{[\%]}\end{array}$ & $\begin{array}{c}\text { Aorta } \\
{[\mathrm{mm}]}\end{array}$ \\
\hline 19 & 42 & 30 & 65 & 73 & 38 \\
\hline 21 & 35 & 22 & 63 & 61 & 35 \\
\hline 22 & 40 & 26 & 72 & 60 & 42 \\
\hline 24 & 72 & 40 & 70 & 60 & 46 \\
\hline 25 & 73 & 53 & 70 & 50 & 50 \\
\multicolumn{5}{l}{$\begin{array}{l}\text { GA - aortic gradient, LVEDd- left ventricular end diastolic diameter, } \\
\text { EF- ejection fraction }\end{array}$}
\end{tabular}

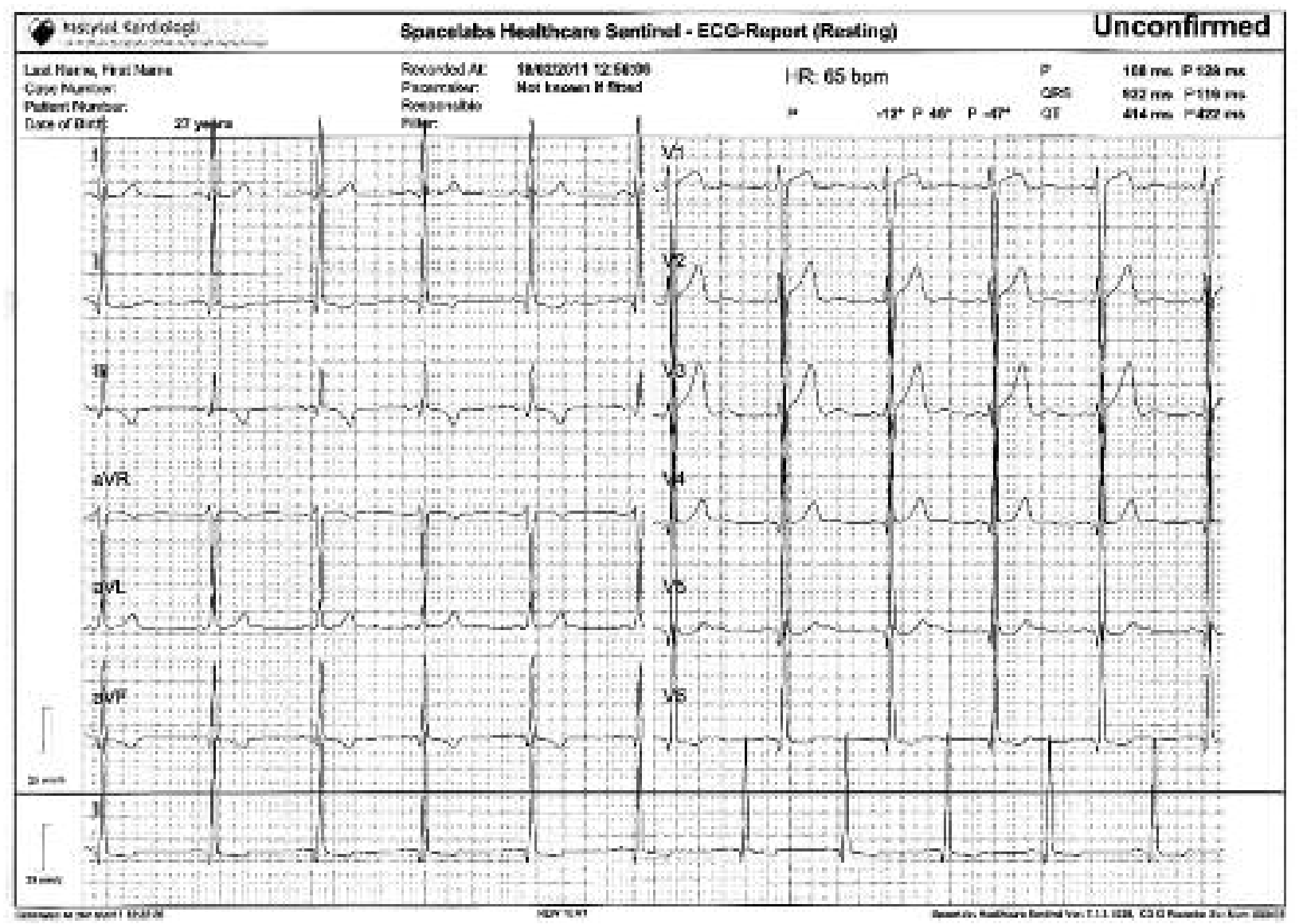

Fig. 1. ECG. Left ventricular hypertrophy (Sokolow index $67 \mathrm{~mm}$ ) and strain (T-wave inversion and biphasic T-waves with ST depression in leads II, III, aVF and V5-V6) 

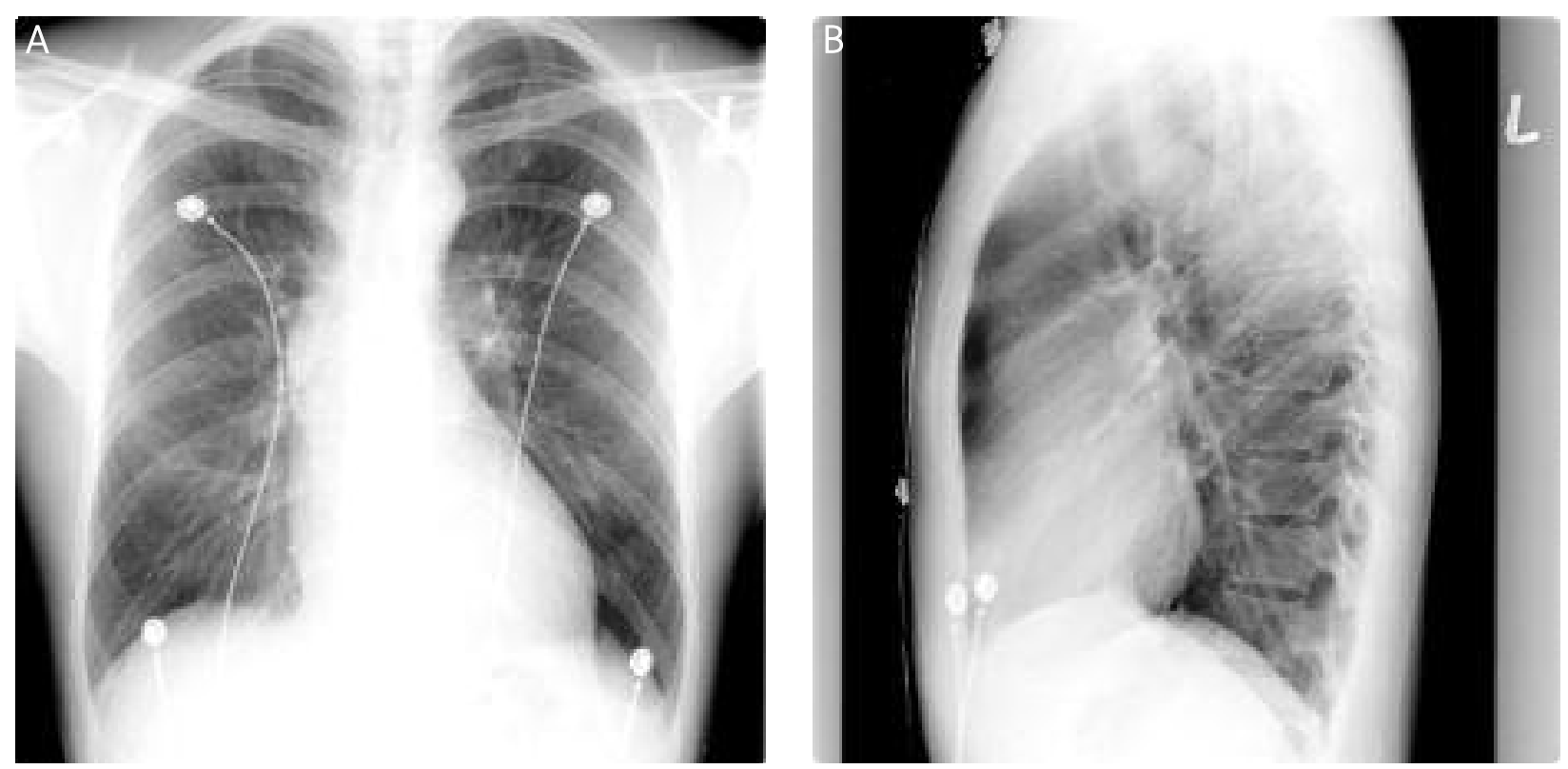

Fig. 2. Chest X-ray. Posterioanterior view (A) and lateral view (B). Mild cardiomegaly with left ventricle enlargement (cardiothoracic ratio 52\%). Dilated ascending aorta
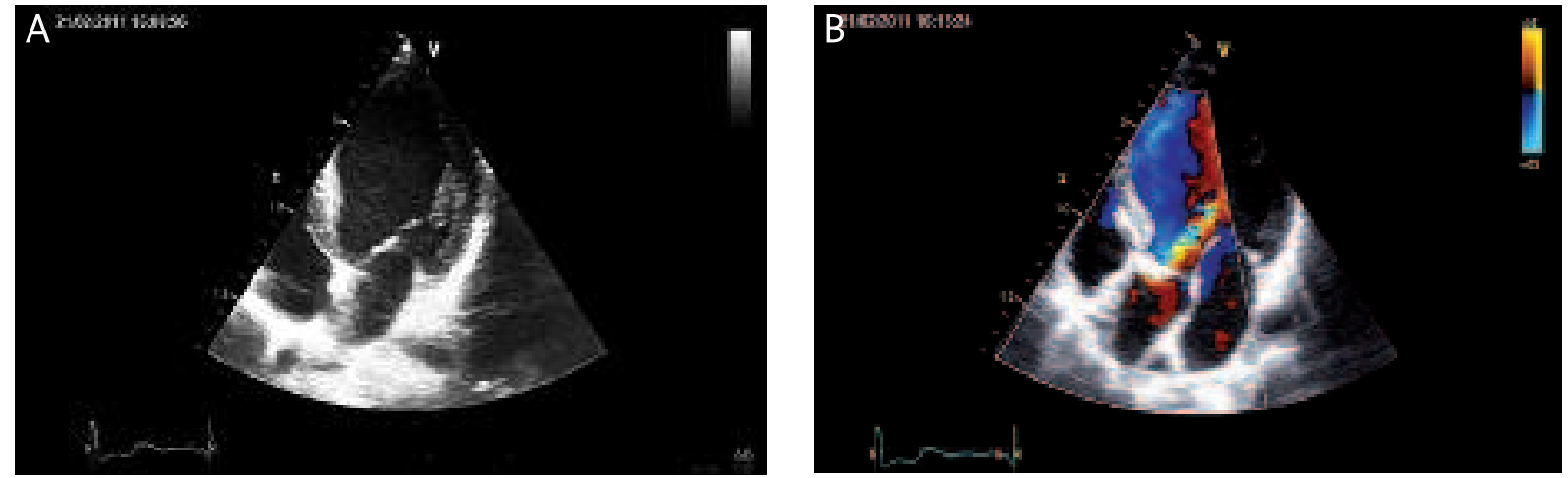

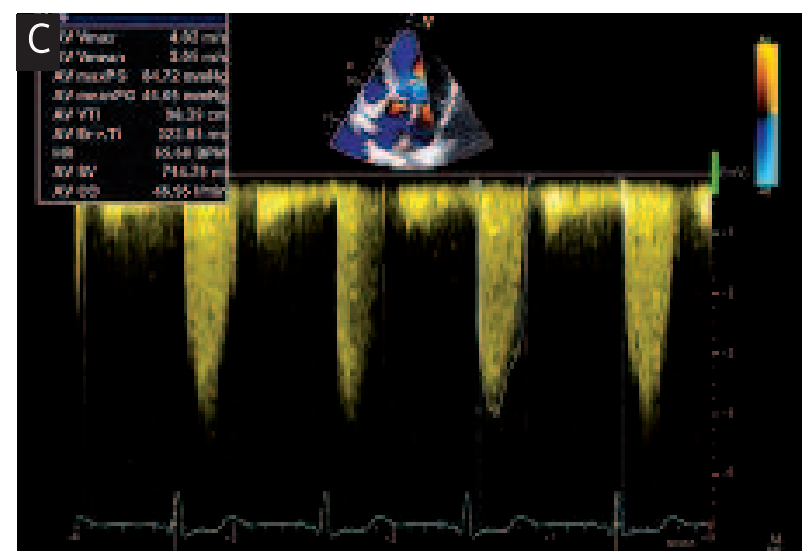

of the disease have been identified. It was found that the abnormal development of the aortic valve and the tendency for calcification of the aortic valve leaflets is caused by mutations in the NOTCH1 gene $[5,6]$. Mutations of the ACTA2 gene cause a contractile dysfunction
Fig. 3. Transthoracic echocardiography. Apical fourchamber view. Enlarged left ventricle (A). Colorcoded Doppler exam. Severe aortic regurgitation (B). Aortic systolic velocity evaluation. Peak gradient is $64 \mathrm{~mm} \mathrm{Hg}$, mean $41 \mathrm{~mm} \mathrm{Hg}$ (C)

of the thin filaments of smooth myocytes of the aorta leading to the development of BAV and TAAD [7]. Both of these mutations have an autosomal dominant mode of inheritance. Two other syndromes which may be accompanied by BAV are also inherited in the same way: Loeys-Dietz 

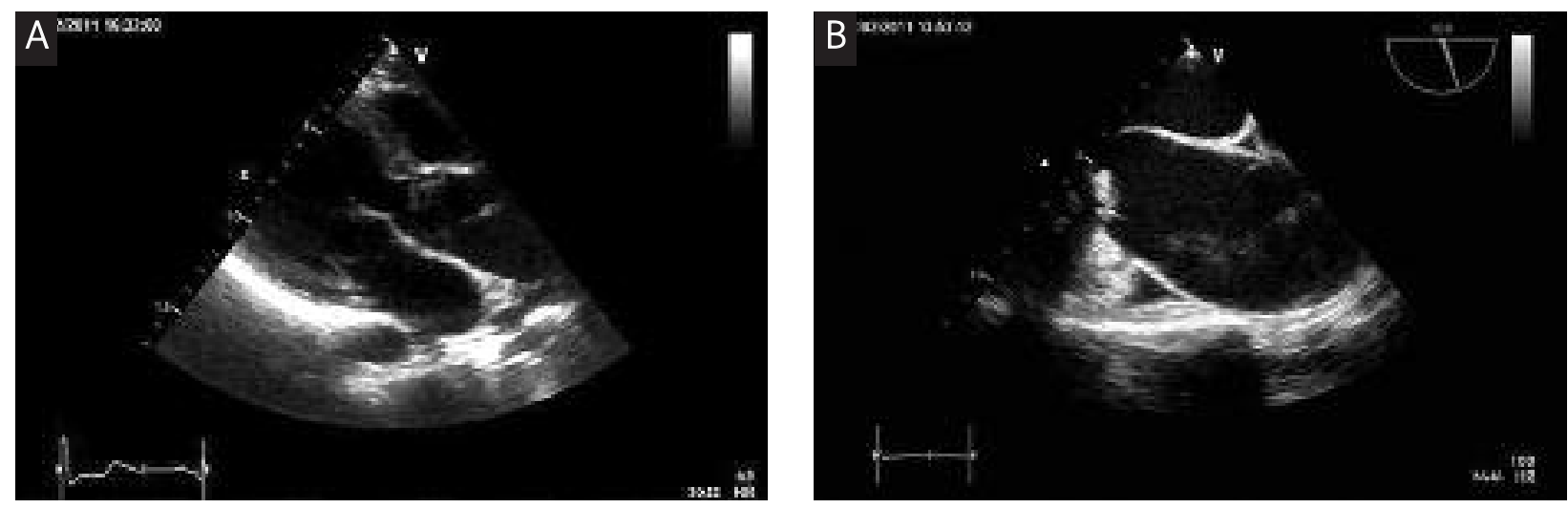

Fig. 4. Transthoracic echocardiography. Parasternal long axis view. Linear echo in the aortic lumen (A). Midesophageal ascending aorta long axis view. Linear echo in the aortic lumen (B)
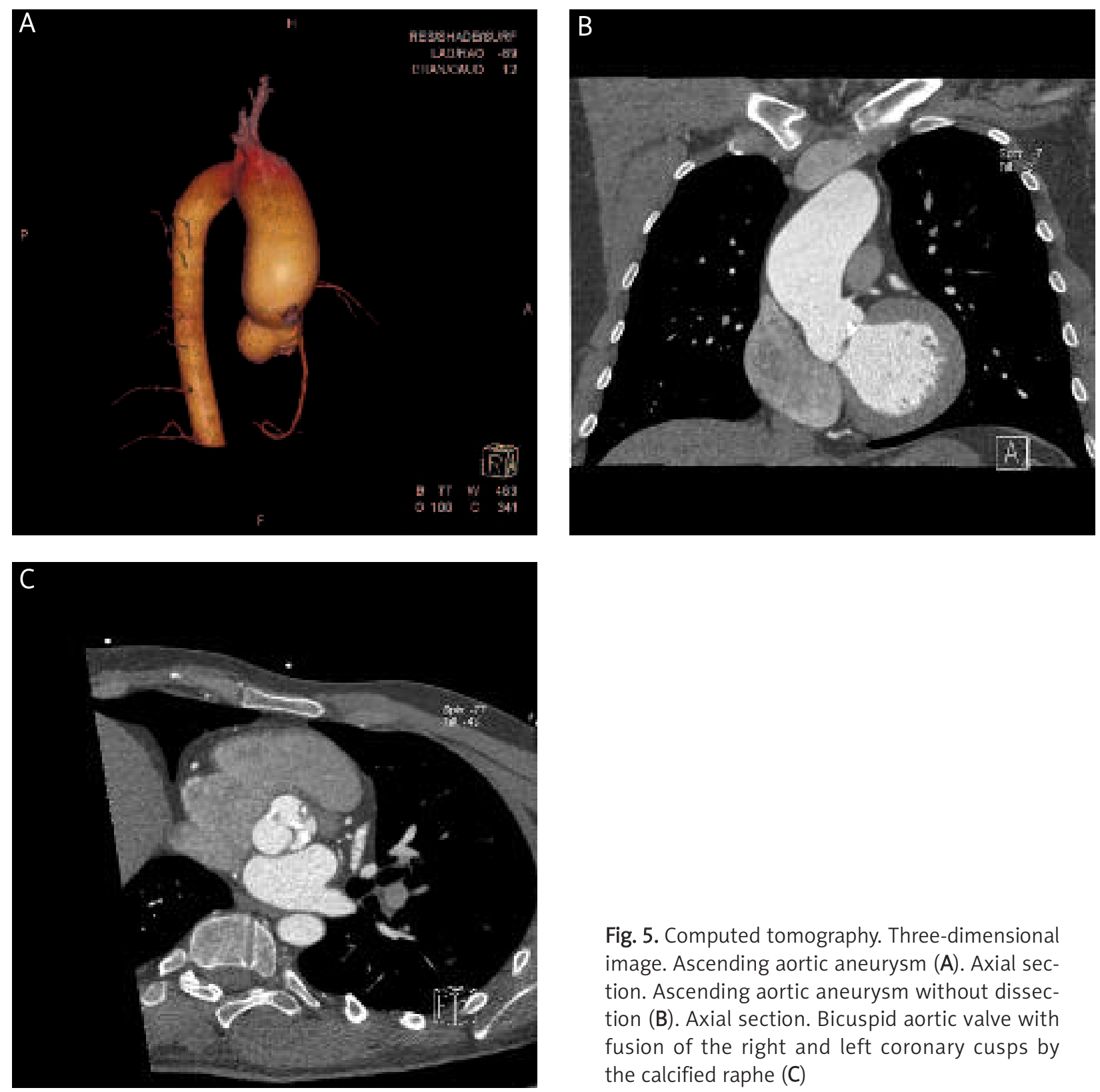

Fig. 5. Computed tomography. Three-dimensional image. Ascending aortic aneurysm (A). Axial section. Ascending aortic aneurysm without dissection (B). Axial section. Bicuspid aortic valve with fusion of the right and left coronary cusps by the calcified raphe (C) 


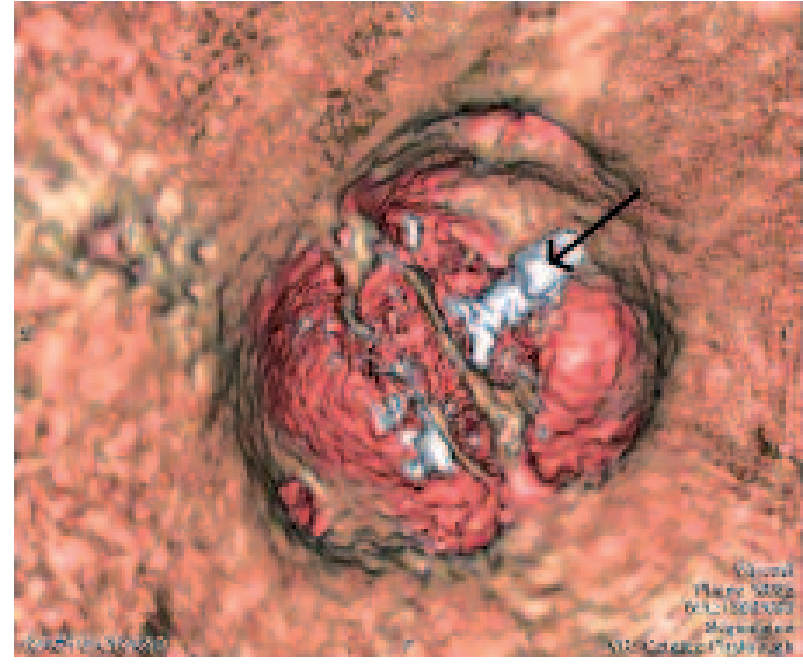

Fig. 6. Computed tomography. Three-dimensional image. Bicuspid aortic valve with fusion of the right and left coronary cusps by the calcified raphe

syndrome (mutations in the TGFBR1 and TGFBR2 genes) and Andersen syndrome (mutations in the KCJN2 gene) $[8,9]$. Other syndromes which can coexist with BAV and which are inherited in an X-linked form include Turner syndrome and X-linked periventricular heterotopia. Another cause of BAV is reduced production of nitric oxide synthase (eNOS) [10].

They most likely lead to distortion of extracellular matrix (ECM) structure. It is caused by improper synthesis, degradation and transport of fibrillin-1, which leads to its deficiency. In the normal tricuspid valve fibrillin-1 binds smooth muscle cells with ECM structures such as elastin and collagen. Fibrillin-1 deficiency causes a lack of support for smooth muscle cells. There is a release of matrix metalloproteinases (MMPs), injury to the ECM including elastin fragmentation, cell death and in consequence the loss of support and flexibility of the aortic wall. These changes in the middle layer of the aortic wall lead to the development and expansion of the aortic aneurysm [1, 4, 11]. The incidence of TAAD in patients with BAV is estimated at about $30-40 \%$, so it is three times higher than in patients with tricuspid valve, in which it is about $12 \%[1,3]$. Aortic dissection is the most dangerous complication of BAV. It occurs in $2-6 \%$ of patients, mostly young and with the presence of BAV regurgitation. It is estimated that aortic regurgitation exists in approximately $20-30 \%$ of patients with $\mathrm{BAV}$, while stenosis is present in $60-70 \%$ of them $[1,4]$.

The main imaging examination used in the diagnosis of BAV is echocardiography. In patients without significant aortic valve dysfunction and with aortic dimension $<40 \mathrm{~mm}$ it is recommended to perform the examination biannually. In patients with significant stenosis/regurgitation of the valve and/or enlarged ascending aorta $\geq 40 \mathrm{~mm}$, the examination should be performed annually.
In our case during 7 years of annual echocardiographic studies there was a gradual mild increase of the aortic valve gradient, left ventricular dilation with its progressive systolic dysfunction and enlargement of the ascending aorta. Due to the presence of symptoms in a patient at the age of 25 years accompanied by significant left ventricular dilation, severe regurgitation and moderate aortic stenosis with ascending aorta enlargement to $52 \mathrm{~mm}$, the patient was qualified for surgery. A comment is required regarding interpretation of the aortic gradient, because of its influence on clinical decision-making. It should be remembered that the transvalvular gradient depends on the stroke volume and in case of its reduction it is helpful to assess the aortic valve area [12]. Due to the high risk of developing TAAD in patients with BAV it is important to assess the aorta.

In our case, both transesophageal and transthoracic echocardiographic examinations showed the presence of additional linear echoes in the aortic lumen, which might have corresponded to ruptured intima. Such echoes require differentiation between dissection and artifact. During the differentiation process it may be helpful to analyze the linear motion of the echo - a chaotic wavy and often flappy motion inconsistent with the cardiac cycle and not parallel to other adjacent structures characterizes ruptured intima. Moreover, in contrast to the artifact, whose intensity decreases gradually in the vessel lumen, the echogenicity of ruptured intima is homogeneous along the entire course. Color-coded Doppler may also be useful in the differentiation of aortic dissection and artifact. In the case of intimal rupture it will show the separation of flow, while the artifact will not affect the distribution of the color-coded signal. It is also worth remembering that an additional echo seen in the aortic lumen may correspond to an overlapping vein running in the proximity of the aorta - it is usually the left brachiocephalic vein. Also in this case Doppler examination is useful in differentiation. It will show the flow on both sides of the linear echo, but the flow in the aorta will have a pulsatile character and the one in the vein will be continuous. Finally, the ruptured intima should be registered in more than one projection $[12,13]$. In the case of diagnostic doubts and the need for detailed assessment of the aneurysm and possible aortic dissection computed tomography should be performed.

The guidelines of European and American societies recommend surgery of the aortic valve in case of severe and symptomatic aortic stenosis/regurgitation or severe and asymptomatic aortic stenosis/insufficiency, when at least one of the following criteria is met: impaired left ventricular systolic function ( $E F<50 \%)$, referral for another type of cardiac surgery (of the aorta, other valves or coronary artery bypass grafting), end-diastolic diameter (EDD) or end-systolic diameter (ESD) of the left ventricle exceeds $70 \mathrm{~mm}$ or $50 \mathrm{~mm}$ respectively (this regards only aortic 
regurgitation), or symptoms appear during the exercise test (this regards only aortic stenosis). The indication for surgical treatment of the aortic aneurysm in patients with BAV is enlargement of the ascending aorta $>50 \mathrm{~mm}$. Replacement of the ascending aorta should also be considered in patients undergoing surgery for the BAV if the diameter of the aorta is $4.5 \mathrm{~cm}$ or more [14-16].

In conclusion, BAV is the most common congenital heart defect. It is often accompanied by valvular dysfunction in the form of stenosis and/or regurgitation. As a result of associated congenital abnormalities of the aortic wall, the defect often coexists with an ascending aortic aneurysm. Therefore, diagnosis of BAV, even without its dysfunction, should be associated with regular evaluation of the valve and the aorta. In addition, it is recommended to perform echocardiographic studies in first-degree relatives of patients with BAV.

\section{References}

1. Siu SC, Silversides CK. Bicuspid aortic valve disease. J Am Coll Cardiol 2010; 55: 2789-2800.

2. Cripe L, Andelfinger G, Martin LJ, et al. Bicuspid aortic valve is heritable. J Am Coll Cardiol 2004; 44: 138-143.

3. Jackson V, Petrini J, Caidahl K, et al. Bicuspid aortic valve leaflet morphology in relation to aortic root morphology: a study of 300 patients undergoing open-heart surgery. Eur J Cardiothorac Surg 2011; 40: 118-124.

4. Davies RR, Kaple RK, Mandapati D, et al. Natural history of ascending aortic aneurysm in the setting of an unreplaced bicuspid aortic valve. Ann Thorac Surg 2007; 83: 1338-1344.

5. Garg V, Muth AN, Ransom JF, et al. Mutations in NOTCH1 cause aortic valve disease. Nature 2005; 437: 270-274.

6. Mohamed SA, Aherrahrou Z, Liptau H, et al. Novel missense mutations (p.T596M and p.P1797H) in NOTCH1 in patients with bicuspid aortic valve. Biochem Res Commun 2006; 345: 1460-1465.

7. Guo DC, Pannu H, Tran-Fadulu V, et al. Mutations in smooth muscle alpha-actin (ACTA2) lead to thoracic aortic aneurysm and dissections. Net Genet 2007; 39: 1488-1493.

8. Loeys BL, Chen J, Neptune ER, et al. A syndrome of altered cardiovascular, craniofacial, neurocognitive and skeletal development caused by mutations in TGFBR1 or TGFBR2. Nat Genet 2005; 37: 275-281.

9. Andelfinger G, Tapper AR, Welch RC, et al. KCNJ2 Mutation results in Andersen syndrome with sex-specific cardiac and skeletal muscle phenotypes. Am J Hum Gen 2002; 71: 663-668.

10. Aicher D, Urbich C, Zeiher A, et al. Endothelial nitric oxide synthase in bicuspid aortic valve disease. Ann Thorac Surg 2007; 83: 1290-1294.

11. Russo CF, Cannata A, Lanfranconi M, et al. Is aortic wall degeneration related to bicuspid aortic valve anatomy in patients with valvular disease? J Thorac Cardiovasc Surg 2008; 136: 937-942.

12. Feigenbaum H, Armstrong WF, Ryan T. Feigenbaum's echocardiography. Lippincott Williams \& Wilkins 2004.

13. Klisiewicz A, Hoffman P, Michałek P, Szymański P. Choroby aorty piersiowej. In: Echokardiografia. [Polish]. Hoffman P, Kasprzak JD (red.). Via Medica, Gdańsk 2004.

14. Hiratzka LF, Bakris GL, Beckamm JA, et al. 2010 ACCF/AHA/AATS/ ACR/ASA/SCA/SCAI/SIR/STS/SVM Guidelines for the Diagnosis and Management of Patients with Thoracic Aortic Disease. J Am Coll Cardiol 2010; 55: 27-129.

15. Baumgartner H, Bonhoeffer P, De Groot Natasja MS, et al. ESC Guidelines for the management of grown-up congenital heart diseases (new version 2010). Eur Heart J 2010; 31: 2915-2957.

16. Vahanian A, Baumgartner H, Bax J, et al. Guidelines on the management of valvular heart disease. The Task Force on the Management of Valvular Heart Disease of the European Society of Cardiology. Eur Heart J 2007; 28: 230-268. 